(c) American Dairy Science Association, 2003.

\title{
Bayesian Estimates of Covariance Components Between Lactation Curve Parameters and Disease Liability in Danish Holstein Cows
}

\author{
J. H. Jakobsen, ${ }^{\star, 1}$ R. Rekaya,† J. Jensen, ${ }^{*}$ D. A. Sorensen, ${ }^{\star}$ P. Madsen, ${ }^{\star}$ \\ D. Gianola, $¥$ L. G. Christensen,§ and J. Pedersen\| \\ *Danish Institute of Agricultural Sciences, Department of Animal Breeding and Genetics, \\ P. O. Box 50, DK-8830 Tjele, Denmark. \\ †University of Georgia, Department of Animal and Dairy Sciences, \\ 106 Animal and Dairy Science Complex, Athens, GA 30602, USA. \\ ‡University of Wisconsin-Madison, Department of Animal Science, \\ 1675 Observatory Drive, WI 53706, USA. \\ $\S R o y a l$ Veterinary and Agricultural University, Department of Animal Science and Animal Health, \\ Grønnegaardsvej 3, DK-1870 Frb. C, Denmark. \\ ||Danish Agricultural Advisory Centre, Udkaersvej 15, Skejby, DK-8200 Aarhus N, Denmark
}

\begin{abstract}
In the present work, covariance components for milk yield and disease liability were estimated with bivariate random regression test-day sire models using a Bayesian approach and implemented via the Gibbs sampler. The data consist of 8075 first-parity Danish Holstein (DH) cows, from 1259 sires, performing in 57 herds from 1992 to 1997. Treatments associated with five different type of diseases were pooled into a single general disease liability for each cow. Two models were fitted to the data. First, using a bivariate model, milk yield is modeled via a random regression, and disease liability via a repeatablility model. Second, using a bivariate model, both milk yield and disease liability are modeled using random regressions. A comparison based on a Bayes factor provides very strong support for the bivariate random regression model.

Posterior means of heritabilities for each of the traits were estimated for five different points in time throughout lactation. Across models, heritabilities for milk yield are lowest in the beginning of the lactation (0.19) and highest at the end of the lactation (0.35). Posterior means of heritabilities of disease liability range from 0.04 to 0.10 for test days, and is equal to 0.20 for the whole lactation. Heritability of persistency measures estimated from the two models are 0.20 and 0.21 . Estimates of posterior means of genetic correlations between single test-day milk yield and single test-day disease liability are in the range of 0.31 to 0.57 . The estimates of posterior mean and of the $95 \%$ posterior
\end{abstract}

\footnotetext{
Received February 3, 2003.

Accepted April 18, 2003.

Corresponding author: J. H. Jakobsen; e-mail: jette.jakobsen@ hgen.slu.se.

${ }^{1}$ Current address: Interbull Centre, Box 7023, SE-750 07 Upp-
} sala, Sweden. interval of the genetic correlation between persistency and (total) disease liability using the model with the highest posterior probability are -0.12 and $(-0.44 ; 0.20)$, respectively. Even though the largest proportion of the posterior probability mass is spread along negative values of the correlation (indicating that individuals with a flatter lactation curve tend to have lower disease liability), a value of zero of the genetic correlation falls comfortably within the $95 \%$ posterior interval. Thus the prospects of reducing incidence of disease by manipulating persistency as defined in this work remain inconclusive.

(Key words: Bayesian analysis, bivariate longitudinal response, test-day yield, threshold model)

Abbreviation key: $\mathbf{D H}=$ Danish Holsteins, $\mathbf{T D}=$ test day.

\section{INTRODUCTION}

Selection for total milk yield has led to considerable increases in milk yield in early and peak lactation. This may have resulted in increased metabolic stress, especially in the period from calving to peak yield. Increased metabolic stress leads to higher predisposition to diseases (Uribe et al., 1995; Pryce et al., 1997). There are two major reasons why a higher susceptibility to disease is not desirable. First, there are ethical concerns related to animal welfare and consumer interests. Second, sick cows are less profitable. In most parts of the world, selection has operated mainly on milk yield in spite of the unfavorable correlated effects on secondary traits (e.g., Christensen, 1998). In the Scandinavian countries, disease resistance has been included in the breeding goal to avoid a further increase of disease susceptibility of dairy cows. Many countries are initiating positive selection pressure on disease resistance by 
incorporating correlated traits such as udder conformation, milkability, and SCC in the selection indices.

Earlier investigations have shown that the genetic correlation between milk yield and disease incidence is unfavorable (Simianer et al., 1991; Uribe et al., 1995; Rupp and Boichard, 1999). However, in these studies, correlations measured associations between total lactation milk yield and disease status within one predefined period of risk.

Use of random regression models (Henderson, Jr., 1982) to analyze test-day (TD) milk yield (Schaeffer and Dekkers, 1994) opens the possibility for better use of data from milk recording systems (Jamrozik et al., 1997; Olori et al., 1999; Rekaya et al., 1999; Lidauer et al., 2000). Use of random regression TD models has enhanced our ability to study time dependent changes in longitudinal data. Genetic correlations between records obtained at different points in time can be estimated using such models. Rekaya et al. (1998) applied random regressions to model disease records observed as binary responses in a number of disease risk periods that formed a longitudinal sequence of binary responses within a lactation. A bivariate approach including milk yield and disease status can disclose genetic and phenotypic associations between these traits and cast light on the role played by the shape of the lactation curve.

Genetic parameters for persistency of lactation (defined below) can be calculated as a byproduct of the estimated covariance parameters in random regression TD models for milk yield (e.g., Jamrozik et al., 1997; van der Linde et al., 2000; Jakobsen et al., 2002). It may be hypothesized that curves with a very high peak in daily milk production in early lactation, followed by a sharp fall in later lactation (less persistent), are associated with metabolic stress and negative energy balance. In contrast, curves with lower peak in early lactation followed by a less sharp fall (more persistent) may result in shorter periods with metabolic load and negative energy balance. Thus, a genetic change towards a flatter lactation curve (more persistent) could be used as a means to lower the disease susceptibility of the dairy cow. In principle, level of peak production in the early period and total production over the period defined by early and late lactation could be relevant parameters to study in this connection. However, in this work, we limit ourselves to a simpler definition of the problem and look at the relative fall in production between early and late lactation. This is defined more precisely below in terms of persistency in the section Functions of Parameters Studied.

The purpose of this investigation is to estimate covariance components for TD milk yields and liability to disease using random regression TD models. More specifically, heritabilities of components of TD milk yield and of liability to disease are estimated as well as genetic correlations between disease liability and: 1) milk yield at different points in time throughout lactation, 2) total 305-d lactation milk yield, and 3) persistency. Two models are fitted to the data using Markov chain Monte Carlo techniques. The models are compared using a Bayes factor (Kass and Raftery, 1995).

\section{MATERIAL AND METHODS}

\section{Data}

A national system for recording of disease treatment in dairy cattle was set up in Denmark in 1990. The service is voluntary and is carried out by a veterinarian. A limited number of farmers participated in the early days, but this number has been increasing steadily with time. Across breeds, the proportion of herds currently participating in the program ranges from 60 to $70 \%$.

Data for the present study, extracted from the national database, covered the period from January 1, 1992, until December 31, 1997. A total of 211 Danish Holstein (DH) herds fulfilled the requirements for disease recording. After deleting herds with nonpurebred animals, with missing TD records, and with fewer than 10 first calvings per year, 57 herds remained. Firstlactation TD production records as well as disease treatments of $\mathrm{DH}$ are included in the analyses presented here. All cows with complete as well as incomplete lactations with at least one TD record are included. Cows with a calving age less than 20 mo or above 39 mo at first calving were deleted. After editing, 8075 firstparity cows remained in the dataset. This comprised 64,994 TD milk records and observations on disease treatments. Observations for daily milk yields range from 2 to $47 \mathrm{~kg}$.

Disease treatments include udder diseases, feet, and leg diseases, digestive diseases, reproductive diseases, and other diseases. In the present study, disease treatments are studied jointly under the heading disease susceptibility. A detailed description of the diseases can be found in Nielsen et al. (1996).

Disease-risk intervals are defined according to number of TD available for the individual cow between $\mathrm{d} 0$ (calving) and $305 \mathrm{~d}$ after calving. The first disease-risk interval is from calving to midpoint between first and second TD after calving, and the last disease risk period is from the midpoint between the last and the second to last TD $305 \mathrm{~d}$ after calving. All other disease-risk intervals are defined from midpoint to midpoint between TD. The binary response is either diseased (1) or nondiseased (0) within the interval in question. For nondiseased cows, the time point corresponding to the observation was the TD for milk recording. For diseased cows, the time point was the day of treatment if only 
one treatment occurred within the interval. With two treatments within the same interval, the time point is defined as the midpoint between the $2 \mathrm{~d}$ of each treatment.

A pedigree file was formed to take account of relationships among the sires of the 8075 cows with data. Even though a sire model was postulated (with additive genetic relationships among sires), for each sire, both male and female ancestors were traced as far back as possible. The total pedigree file contains 5110 animals, of which 1259 sires have progeny in the data. Only 16 sires have more than 100 daughters with records; 1167 sires have 10 or less daughters with records.

\section{Models}

The following model [1] is applied to milk yield and to disease liability:

$$
\begin{gathered}
\mathrm{Y}_{i j k l m}=\operatorname{HTD}_{i}+\mathrm{AC}_{j}+\sum_{o=1}^{q} b_{j o} z_{m o}+\sum_{o=1}^{q} s_{k o} z_{m o} \\
+\sum_{o=1}^{q} p_{l o} z_{m o}+\mathrm{e}_{i j k l m}
\end{gathered}
$$

where

$$
\begin{aligned}
\mathrm{Y}_{i j k l m}= & \text { observation of TD record } m \text { of milk or disease } \\
& \text { liability of cow } l \text {, of the } k \text { th sire, calved in the } \\
& j \text { th age class, in the } i \text { th herd-test-date; } \\
\mathrm{HTD}_{i}= & \text { fixed effect of the } i \text { th herd-test-date }(i=1, \ldots, \\
& 3659) ; \\
\mathrm{AC}_{j}= & \text { fixed effect of the } j \text { th age at calving }(j=1, \ldots, \\
& 20) ; \\
\mathrm{q}= & \text { number of covariates describing the shape of } \\
& \text { the lactation curve; } \\
b_{j o}= & \text { fixed regression coefficient for } \mathrm{z}_{m o} ; \\
\mathrm{z}_{m o}= & \text { oth covariate associated with DIM; } \\
s_{k o}= & \text { sire random regression coefficient; } \\
p_{l o}= & \text { permanent environmental random regression } \\
& \text { coefficient; } \\
\mathrm{e}_{i j k l m}= & \text { random residual corresponding to } \mathrm{Y}_{i j k l m .} .
\end{aligned}
$$

Two different models, which are special cases of [1], are postulated. In both cases, the submodel for production is held constant. Several different functions have been applied in random regression models (e.g., Van der Werf et al., 1998; Brotherstone et al., 2000; Schaeffer et al., 2000). Here a third-order normalized Legendre polynomial (Kirkpatrick et al., 1990) was used in the fixed part as well as in the random additive genetic and the permanent environmental parts of the model for production. The third-order Legendre polynomial can be written as:

$$
\begin{aligned}
\mathrm{L} 4=\gamma_{0} & +\gamma_{1} \sqrt{\frac{3}{2}} k+\gamma_{2}\left(\sqrt{\frac{45}{8}} k^{2}-\sqrt{\frac{5}{8}}\right) \\
& +\gamma_{3}\left(\sqrt{\frac{175}{8}} k^{3}-\sqrt{\frac{63}{8}} k\right),
\end{aligned}
$$

where $\gamma_{0}, \gamma_{1}, \gamma_{2}$, and $\gamma_{3}$ are regression coefficients that are denoted $b_{j o}, s_{k o}$, and $p_{l o}$ in [1]. Finally $k=-1+$ $\frac{2}{300} \times(D I M-5)$, which means that the polynomial is defined in the period from d 5 to 305 after calving. Further details can be found in Jakobsen et al. (2002).

For disease liability, two different submodels were postulated. The first one is a repeatability model (a zero order polynomial). This model was labelled [1.1]. In the second model, a Legendre polynomial of order two was used in the fixed, in the additive genetic, and in the permanent environmental parts. This model is labeled [1.2]. The second-order polynomial is defined in the period from d 1 to 305 after calving and, therefore, $k$ is calculated as $k=-1+\frac{2}{304} \times(D I M-1)$.

\section{Statistical Analyses, Implementation and Model Comparison}

A threshold model (Wright, 1934) was used to map an assumed underlying continuous disease liability to the two observed disease classes. A linear random regression bivariate sire model is fitted to milk yield and the underlying disease liability using Gaussian assumptions. In matrix notation the bivariate model can be written as

$$
\begin{aligned}
{\left[\begin{array}{l}
\mathbf{Y} \\
l
\end{array}\right]=} & {\left[\begin{array}{cc}
\mathbf{X}_{1} & \mathbf{0} \\
\mathbf{0} & \mathbf{X}_{2}
\end{array}\right]\left[\begin{array}{l}
\mathbf{b}_{1} \\
\mathbf{b}_{2}
\end{array}\right]+\left[\begin{array}{cc}
\mathbf{Z}_{1} & \mathbf{0} \\
\mathbf{0} & \mathbf{Z}_{2}
\end{array}\right]\left[\begin{array}{l}
\mathbf{s}_{1} \\
\mathbf{s}_{2}
\end{array}\right] } \\
& +\left[\begin{array}{cc}
\mathbf{Z}_{1} & \mathbf{0} \\
\mathbf{0} & \mathbf{Z}_{2}
\end{array}\right]\left[\begin{array}{l}
\mathbf{p}_{1} \\
\mathbf{p}_{2}
\end{array}\right]+\left[\begin{array}{l}
\mathbf{e}_{1} \\
\mathbf{e}_{2}
\end{array}\right]
\end{aligned}
$$

where $\mathbf{Y}$ is the vector of TD production records, $l$ is the vector of disease liabilities, $\mathbf{b}_{1}, \mathbf{b}_{\mathbf{2}}, \mathbf{s}_{\mathbf{1}}, \mathbf{s}_{\mathbf{2}}, \mathbf{p}_{\mathbf{1}}, \mathbf{p}_{\mathbf{2}}$, are vectors of location parameters of "fixed" effects, sire effects and permanent environmental effects, respectively, and $\mathbf{X}_{1}, \mathbf{X}_{2}, \mathbf{Z}_{1}, \mathbf{Z}_{2}$ are the corresponding incidence matrices. Vectors $\mathbf{e}_{1}$ and $\mathbf{e}_{2}$ contain random residuals for test day milk yield and disease liability.

To simplify notation we write 


$$
\begin{gathered}
\mathbf{U}=\left[\begin{array}{c}
\mathbf{Y} \\
l
\end{array}\right], \mathbf{X}=\left[\begin{array}{ll}
\mathbf{X}_{1} & \mathbf{0} \\
\mathbf{0} & \mathbf{X}_{2}
\end{array}\right], \mathbf{b}=\left[\begin{array}{l}
\mathbf{b}_{1} \\
\mathbf{b}_{2}
\end{array}\right], \mathbf{Z}=\left[\begin{array}{cc}
\mathbf{Z}_{1} & \mathbf{0} \\
\mathbf{0} & \mathbf{Z}_{2}
\end{array}\right], \\
\mathbf{s}=\left[\begin{array}{l}
\mathbf{s}_{1} \\
\mathbf{s}_{2}
\end{array}\right], \mathbf{p}=\left[\begin{array}{l}
\mathbf{p}_{1} \\
\mathbf{p}_{2}
\end{array}\right] \text { and } \mathbf{e}=\left[\begin{array}{l}
\mathbf{e}_{1} \\
\mathbf{e}_{2}
\end{array}\right]
\end{gathered}
$$

A fully Bayesian analysis is conducted and the conditional sampling model for the observed data and liabilities is assumed to be

$\mathbf{U} \mid \mathbf{b}, \mathbf{s}, \mathbf{p}, \mathbf{G}_{\mathbf{0}}, \mathbf{P}_{\mathbf{0}}, \mathbf{R} \sim \operatorname{MVN}\left(\mathbf{X b}+\mathbf{Z} \mathbf{s}+\mathbf{Z} \mathbf{p},\left(\mathbf{R}_{\mathbf{0}} \otimes \mathbf{I}\right)\right)$.

Assumed prior distributions of the parameters of the model are:

$\mathbf{b} \propto$ constant

$\mathbf{s} \mid \mathbf{G}_{\mathbf{0}} \sim \operatorname{MVN}\left[\mathbf{0},\left(\mathbf{G}_{\mathbf{0}} \otimes \mathbf{A}\right)\right]$,

$\mathbf{p} \mid \mathbf{P}_{\mathbf{0}} \sim \operatorname{MVN}\left[\mathbf{0},\left(\mathbf{P}_{\mathbf{0}} \otimes \mathbf{I}_{\mathbf{N A}}\right)\right]$,

$\mathbf{G}_{\mathbf{0}} \mid \mathbf{S}_{\mathbf{g}}, \nu_{\mathrm{g}} \sim \mathrm{IW}_{\mathrm{j}}\left[\mathbf{S}_{\mathbf{g}} \nu_{\mathrm{g}}, \nu_{\mathrm{g}}\right]$

$\mathbf{P}_{\mathbf{0}} \mid \mathbf{S}_{\mathbf{p e}}, \nu_{\mathrm{pe}} \sim \mathrm{IW}_{\mathrm{j}}\left[\mathbf{S}_{\mathbf{p e}} \nu_{\mathrm{pe}}, \nu_{\mathrm{pe}}\right]$,

$\mathbf{R}_{\mathbf{0}} \mid \mathbf{S}_{\mathbf{R}}, \nu_{\mathrm{R}} \sim \mathrm{CIW}_{2}\left[\mathbf{S}_{\mathbf{R}} \nu_{\mathrm{R}}, \nu_{\mathrm{R}}\right]$.

In these expressions, MVN[.,.] is the multivariate normal distribution. $\mathrm{IW}_{\mathrm{j}}[., .$,$] is the j x j$ inverse Wishart distribution, where $j$ is the dimension of the genetic and permanent environmental covariance matrices $\mathbf{G}_{\mathbf{0}}$ and $\mathbf{P}_{\mathbf{0}}$, respectively. $\mathrm{CIW}_{2}$ is the conditional inverse Wishart distribution of dimension 2, equal to the dimension of $\mathbf{R}_{\mathbf{0}}$, given the residual variance for the underlying disease liability. This variance is set equal to 1 to achieve identifiability in the likelihood. $\mathbf{A}$ is the relationship matrix, $\mathbf{I}_{\mathbf{N A}}$ is the identity matrix of dimension NA, where NA is number of animals with data, and $\mathbf{S}_{\mathbf{g}}$, $\nu_{\mathrm{g}}, \mathbf{S}_{\mathbf{p e}}, \nu_{\mathrm{pe}}, \mathbf{S}_{\mathbf{R}}, \nu_{\mathrm{R}}$, are prior values and degrees of belief for additive genetic, permanent environmental and residual covariances, respectively. Degrees of belief were chosen to represent vague a priori information.

The Bayesian models were fitted using a Gibbs sampling algorithm based on a single long chain. In the context of the models postulated, the fully posterior distributions were either multivariate normal or scale inverted Wishart. Details are provided in Jakobsen (2000). A chain length equal to 150,000 was used, and the first 30,000 samples were discarded (burn in). This leads to estimates of the posterior means reported with coefficients of variation due to Monte Carlo error smaller than $1.5 \%$.

The two models are compared via a Bayes factor (Kass and Raftery, 1995). Briefly, the Bayes factor $\mathrm{B}_{i j}$, is the ratio between the posterior odds of model $i$ and model $j$, and the prior odds. It provides a measure of whether the data have increased or decreased the odds on model $i$, relative to model $j$. The interpretation of a Bayes factor equal to 100 , say, is that the observed data are 100 times more likely to have been generated under model $i$ than under model $j$. Here, Bayes factors are computed as a byproduct of the Gibbs sampler, using the Monte Carlo consistent estimator proposed by Newton and Raftery (1994). Details specific to the present study are shown in Jakobsen (2000).

\section{Functions of Parameters Studied}

Persistency. Persistency is defined as the linear slope between yields at d 60 and 280. A large negative value indicates low persistency, whereas a flatter lactation curve results in a larger (less negative) value of the slope and a relatively higher persistency. This persistency measure does not account for differences in yield in the period between 60 and 280 after calving. It was first described by Jamrozik et al. (1998), who used it with opposite sign.

Heritabilities. Heritability $\left(h_{i(t)}^{2}\right)$ for TD $(i)$ of trait $(t)$ (milk yield or disease liability) is defined as:

$$
h_{i(t)}^{2}=\frac{4 \times \mathbf{z}_{i(t)}^{\prime} \mathbf{G}_{(t)} \mathbf{z}_{i(t)}}{\mathbf{z}_{i(t}^{\prime} \mathbf{G}_{(t)} \mathbf{z}_{i(t)}+\mathbf{z}_{i(t)}^{\prime} \mathbf{P}_{(t)} \mathbf{z}_{i(t)}+k \times \sigma_{e(t)}^{2}},
$$

where $\mathbf{z}_{\mathbf{i}(t)}$ is a vector of covariates for trait $t$ at TD $i$, $\mathbf{G}_{(t)}$ and $\mathbf{P}_{(t)}$ are the parts of the estimated covariance matrices corresponding to the specific trait $(t)$ for genetic and permanent environmental effects and $\sigma_{e(t)}^{2}$ is the residual variance of trait $t . k$ is a constant that takes the values 1,305 , and 2 , if the heritability is calculated for $1 \mathrm{~d}$, for a 305-d period, and for persistency, respectively. The symbol $i$ represents a day (day), a 305-d yield (305- $d$ ) and persistency (pers), and the corresponding $\mathbf{z}_{i(t)}$ were calculated as $\mathbf{z}_{d a y(t)}=\mathbf{z}_{d a y_{i}(t)}$, where $j$ is the symbol for one specific day, $\mathbf{z}_{305-d(t)}=$ 305

$\sum_{j=1}^{305} \mathbf{z}_{d a y_{\mathrm{i}}}(t)$, and $\mathbf{z}_{p e r s}=\mathbf{z}_{d a y_{60}}-\mathbf{z}_{d a y_{280}}$.

Genetic correlations. Genetic correlation between milk $(m)$ and disease liability $(d)$ observed at day $i$ and day $j$, respectively, is defined as:

$$
\boldsymbol{r}_{g(i, j)}=\frac{\mathbf{z}_{m(i)}^{\prime} \mathbf{G}_{(m, d)} \mathbf{z}_{d(j)}}{\sqrt{\mathbf{z}_{m(i)}^{\prime} \mathbf{G}_{(m, m)} \mathbf{z}_{m(i)} \times \mathbf{z}_{d(j)}^{\prime} \mathbf{G}_{(d, d)} \mathbf{z}_{d(j)}}}
$$

where $\mathbf{z}_{m(i)}$ and $\mathbf{z}_{d(j)}$ are vectors of covariates for TD $i$ and trait $m$, and for TD $j$ and trait $d$, respectively. $\mathbf{G}_{(m, d)}$, $\mathbf{G}_{(m, m)}, \mathbf{G}_{(d, d)}$ are the parts of the genetic covariance matrix containing genetic covariances between curve parameters for milk and disease liability, genetic co- 


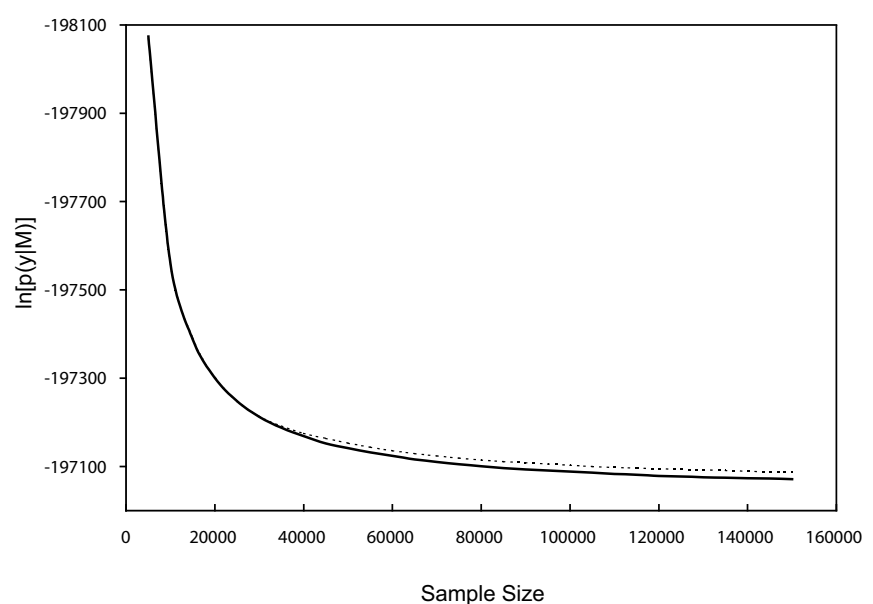

Figure 1. Average logarithm of integrated likelihoods as a function of sample size for model 1.1 (dotted line) and model 1.2, respectively.

variances for curve parameters for milk, and genetic covariances for curve parameters for disease liability, respectively. For calculation of correlations comprising 305-d yield and persistency, $\mathbf{z}_{m(i)}$ and $\mathbf{z}_{d(j)}$ can be replaced by $\mathbf{z}$-values for 305-d yield and persistency. Values of $\mathbf{z}$ were calculated as shown in the Section for heritabilities.

\section{RESULTS}

\section{Model Comparison}

The Newton-Raftery estimates of the logarithm of the integrated likelihoods were equal to $-198,407$ and $-198,379$ for models [1.1] and [1.2], respectively. If one assigns equal a priori probabilities to the models, this means that model [1.2] is $\exp (28)$ more probable a posteriori than model [1.1]. Figure 1 shows Monte Carlo estimates of the logarithm of the integrated likelihoods computed from samples that increase in size in steps of one, from 1 to 150,000 . The figure indicates that the ranking of the models stabilize to a consistent pattern when sample size reaches 40,000 and that the Monte Carlo estimators show little change when sample size is larger than 100,000 .

\section{Posterior Mean of Heritabilities}

Monte Carlo estimates of posterior mean of heritabilities for single TD milk yields and for 305-d milk production are shown in Table 1 for models [1.1] and [1.2]. In both cases, the submodel for milk yield is the same, whereas the order of fit for the regressions in the submodel for disease liability varies between models. The estimates obtained from the two models are very similar.
Table 1. Posterior mean of heritabilities for single test days, total yield, and persistency for the two traits milk yield and disease liability for models [1.1] and [1.2]. ${ }^{1}$

\begin{tabular}{lll}
\hline & \multicolumn{2}{c}{ Model } \\
\cline { 2 - 3 } & 1.1 & 1.2 \\
\hline Milk yield & & \\
$h_{\text {day } 5}^{2}$ & 0.19 & 0.20 \\
$h_{\text {day } 25}^{2}$ & 0.23 & 0.24 \\
$h_{\text {day } 85}^{2}$ & 0.29 & 0.30 \\
$h_{\text {day } 165}^{2}$ & 0.30 & 0.30 \\
$h_{\text {day } 285}^{2}$ & 0.34 & 0.35 \\
$h_{\text {tot }}^{2}$ & 0.38 & 0.39 \\
$h_{\text {pers }}^{2}$ & 0.20 & 0.21 \\
Disease liability & & \\
$h_{\text {day5 }}^{2}$ & $0.20^{2}$ & 0.04 \\
$h_{\text {day } 25}^{2}$ & $0.20^{2}$ & 0.06 \\
$h_{\text {day } 85}^{2}$ & $0.20^{2}$ & 0.10 \\
$h_{\text {day165 }}^{2}$ & $0.20^{2}$ & 0.06 \\
$h_{\text {day } 285}^{2}$ & $0.20^{2}$ & 0.06 \\
$h_{\text {tot }}^{2}$ & 0.20 & 0.20 \\
\hline
\end{tabular}

${ }^{1}$ Posterior standard deviations of heritabilities were in the range 0.01 to 0.06

${ }^{2}$ The repeatability model assumes constant heritability across time.

Estimates of posterior means of heritability for persistency were 0.20 and 0.21 , from models [1.1] and [1.2], respectively. The $95 \%$ posterior probability intervals for both models were $(0.12,0.29)$, which is indicative of sharp posterior distributions.

Estimates of posterior means of heritabilities for liability to disease at d 5, 25, 85, 165, 285, and summed over $305 \mathrm{~d}$ are shown in Table 1 for models [1.1] and [1.2]. The estimate based on model [1.1], which for liability to disease is a repeatability model, is 0.20 . Inferences based on model [1.2], resulted in estimates of posterior means of heritabilities for disease of 0.04 , $0.06,0.10,0.06$, and 0.06 for d 5, 25, 85, 165, and 285, respectively. The estimate of the posterior mean of heritability summed over a complete lactation is 0.20 , with a Monte Carlo estimate of the $95 \%$ posterior interval equal to $(0.12 ; 0.31)$.

\section{Posterior Mean of Genetic Correlations}

Estimates of posterior means of genetic correlations between milk yield and disease liability for all combinations of DIM 5, 25, 85, 165, 285, total 305-d yield, and persistency are shown in Table 2 and Table 3 for model [1.1] and [1.2], respectively. The posterior means of the genetic correlations between single TD of milk production and disease liability are in the range 0.26 to 0.57 .

Estimates of posterior means of the genetic correlation between 305-d milk yield and total disease liability 
Table 2. Posterior mean of genetic correlations for single test days, 305-d yield, and persistency for milk yield and disease liability; model [1.1]. ${ }^{1}$

\begin{tabular}{lrrrrrrr}
\hline & \multicolumn{7}{c}{ Milk yield } \\
\cline { 2 - 8 } Disease liability & $\mathrm{d} 5$ & $\mathrm{~d} 25$ & $\mathrm{~d} 85$ & $\mathrm{~d} 165$ & $\mathrm{~d} 285$ & 305-d & pers \\
\hline Any day in lactation & 0.35 & 0.42 & 0.43 & 0.39 & 0.50 & 0.47 & -0.19 \\
305-d & -0.11 & -0.06 & -0.01 & -0.05 & 0.13 & 0.47 & -0.19 \\
\hline
\end{tabular}

${ }^{1}$ Posterior standard deviations of genetic correlations were in the range 0.09 to 0.14 (except for second row, d 85 and 165 whose values were 0.04 and 0.03 , respectively).

are 0.47 and 0.52 , depending on the model used in the analysis. Posterior means of genetic correlations between 305-d milk production and disease liability at d $5,25,85,165$, and 285 , respectively, are in the range 0.25 to 0.57 and are close to the correlation estimates between single TD of milk production and disease liability.

Estimates of posterior means of the genetic correlations between disease liability summed over the complete lactation and milk yield at d 5, 25, 85, 165, and 285, respectively, are also shown in Table 2 and Table 3. For model [1.1] correlations are close to 0 , whereas for model [1.2], correlations are in the same range as between total 305-d milk production and individual days of disease liability.

Estimates of posterior means of genetic correlations between persistency and 305-d milk yield are unfavorable (-0.21 and -0.24 from model [1.1] and [1.2], respectively), indicating an antagonistic relationship between persistency and total milk yield. In other words, an increase in milk production leads to a decrease in persistency, on average (a steeper negative slope of the lactation curve). However, the Monte Carlo estimates of the $95 \%$ posterior intervals are $(-0.75 ; 0.33)$ and $(-0.77$; 0.35), for model [1.1] and [1.2], respectively, reflecting considerable posterior uncertainty.

Estimates of posterior means of genetic correlations between persistency and total disease liability are also shown in Tables 2 and 3. The estimates of the posterior mean and of the $95 \%$ posterior interval based on model [1.1] are -0.19 and $(-0.47 ; 0.09)$, respectively. The cor- responding estimates obtained from model [1.2], are -0.12 and $(-0.44 ; 0.20)$. Even though the largest proportion of the probability mass is spread along negative values of the correlation (indicating that a steeper negative slope-i.e., less persistency, is associated with higher disease liability), especially for the best fitting model (model 1.2), a value of zero for the genetic correlation falls within the $95 \%$ posterior interval. The disclosed association is weak.

\section{DISCUSSION}

The central theme in this work was to investigate a possible genetic association between disease liability, in general, and the shape of the lactation curve. Other related issues in this work involve inferences about the genetic correlation between the form of the lactation curve and measures of milk production, as well as inferences about heritability for measures of milk production and for disease liability.

The heritability estimates for TD milk yields, apart from the estimates for d 285, are very similar to the REML estimates obtained by Jakobsen et al. (2002) using the same data, but slightly higher than those obtained by Lidauer and Mäntysaari (1999), and slightly lower than estimates reported by Firat et al. (1997). In contrast with these studies, we found that the genetic variance for milk yield increases at the end of the lactation.

Estimates of posterior means of heritability of 0.20 and 0.21 for persistency obtained in the present study

Table 3. Posterior mean of genetic correlations for single test days, 305-d yield, and persistency for milk yield and disease liability; model [1.2].

\begin{tabular}{lllllllr}
\hline \multirow{2}{*}{$\begin{array}{l}\text { Disease } \\
\text { liability }\end{array}$} & $\mathrm{d} \mathrm{5}$ & $\mathrm{d} 25$ & $\mathrm{~d}$ 85 & $\mathrm{d} \mathrm{165}$ & $\mathrm{d} 285$ & 305-d & pers \\
\cline { 2 - 8 } & $0.46^{1}$ & 0.44 & 0.48 & 0.45 & 0.46 & 0.25 & 0.07 \\
d 5 & 0.46 & 0.38 & 0.34 & 0.38 & 0.31 & 0.40 & -0.01 \\
d 25 & 0.38 & 0.43 & 0.43 & 0.42 & 0.38 & 0.45 & -0.04 \\
d 85 & 0.43 & 0.26 & 0.40 & 0.42 & 0.39 & 0.46 & -0.06 \\
d 165 & 0.42 & 0.37 & 0.52 & 0.51 & 0.57 & 0.57 & -0.17 \\
d 285 & 0.41 & 0.47 & 0.49 & 0.46 & 0.49 & 0.52 & -0.12 \\
305-d &
\end{tabular}

${ }^{1}$ Posterior standard deviations of genetic correlations were in the range 0.13 to 0.18 . 
are in good agreement with the figures 0.15 and 0.30 reported by Jamrozik et al. (1998) and van der Linde et al. (2000), respectively.

Heritability estimates for disease liability in complete lactation were 0.20 using both models. This estimate is similar to that reported by Heringstad et al. (1999), and slightly higher than the estimate of 0.15 in Uribe et al. (1995); both studies involved mastitis data and used a model with one disease risk period only.

Inferences about the genetic correlation between disease liability and persistency on the one hand, as well as between measures of milk production and persistency on the other, are unfortunately diffuse. A value of zero of the genetic correlations fall within the $95 \%$ posterior intervals. Notwithstanding, in both cases, the largest proportion of the posterior uncertainty is spread along negative values of the correlation. This indicates that cows with flatter lactation curves tend to be less liable to disease and that an increase in milk production tends to lower persistency.

The estimates of the genetic correlation between single TD milk production and single TD disease liability are in the range 0.26 to 0.57 , with posterior standard deviations ranging from 0.10 to 0.16 . Similar figures are obtained for 305-d milk yield and 305-d disease liability. This positive (unfavorable) association has also been reported in other studies using one disease risk period only (Simianer et al., 1991; Uribe et al., 1995; Rupp and Boichard, 1999).

It is well known that the shape of the lactation curve is flatter for first-parity cows compared with cows of later parities (e.g., Leukkunen, 1985). Also, as noted by Schaeffer et al. (2000), estimates of covariance components across parities for random regression curve parameters, and thus persistency, are different. This is further supported by the work of Jamrozik et al. (1998). These authors estimated genetic correlations between persistency in first and second lactation, first and third lactation, and second and third lactation. Their point estimates are $0.37,0.31$, and 0.60 , respectively. These figures suggest that persistency in different lactations can be considered as different traits. In the present study, disease liability and persistency were studied for first-parity data only. It seems relevant to investigate genetic associations between disease and measures of persistency in later parities, where disease frequency tends to be higher.

In hindsight, what could have been done to obtain a deeper understanding of the issues involved in this study? First, the definition of persistency used in this work does not account for the actual level of production in early and late lactation. It does not include total production in the relevant period either. These can be important factors to take into account in studies of pos- sible associations between production and metabolic stress. Further, one cannot dismiss the possibility that other approaches of incorporating information on the form of the lactation curve may lead to more useful results in terms of reducing incidence of disease (i.e., utilizing all the information in the genetic covariance matrix associating curve parameters and disease liability). Secondly, there is the issue of the definition of disease liability. Different diseases have different prevalence in lactation periods and may vary in the way they are genetically and environmentally associated with production. Finally, only the association between disease and milk yield was studied here. Other traits such as protein and fat yield could be more relevant.

Despite these reservations, the pattern of genetic variation and covariation that emerges from our analysis confirms that disease liability is unfavorably associated with general measures of yield, that yield tends to be unfavorably associated with persistency, and that persistency measured in first lactation shows a weak unfavorable relationship with disease liability. This latter inference is associated with high posterior uncertainty.

\section{ACKNOWLEDGMENTS}

We are grateful to two anonymous referees whose comments significantly improved the revised version of this manuscript.

\section{REFERENCES}

Brotherstone, S., I. M. S. White, and K. Meyer. 2000. Genetic modelling of daily milk yield using orthogonal polynomials and parametric curves. Anim. Sci. 70:407-415.

Christensen, L. G. 1998. Possibilities for genetic improvement of disease resistance, functional traits and animal welfare. Acta Agric. Scand. Sect. A. Animal Sci. 1998. Suppl. 29:77-89.

Firat, M. Z., C. M. Theobald, and R. Thompson. 1997. Univariate analysis of test day milk yields of British Holstein-Friesian heifers using Gibbs sampling. Acta Agric. Scand. Sect. A, Anim. Sci. 47:213-220.

Henderson Jr., C. R. 1982. Analysis of covariance in mixed models: Higher-level, nonhomogeneous, and random regressions. Biometrics 38:623-640.

Heringstad, B., R. Rekaya, D. Gianola, G. Klemetsdal, and K. A. Weigel. 1999. Bayesian analysis of heritability of liability to clinical mastitis in Norwegian cattle with a threshold model. Page 59 in 50th Ann. Mtg. EAAP, Zurich, Switzerland.

Jakobsen, J. H. 2000. Genetic correlations between the shape of the lactation curve and disease resistance in dairy cattle. Ph.D. Thesis, The Royal Veterinary and Agricultural University, Denmark.

Jakobsen, J. H., P. Madsen, J. Jensen, J. Pedersen, L. G. Christensen, and D. Sorensen. 2002. Genetic parameters for milk production and persistency for Danish Holsteins estimated in random regression models using REML. J. Dairy Sci. 85:1607-1616.

Jamrozik, J., L. R. Schaeffer, and J. C. M. Dekkers. 1997. Genetic evaluation of dairy cattle using test day yields and random regression model. J. Dairy. Sci. 80:1217-1226.

Jamrozik, J., G. Jansen, L. R. Schaeffer, and Z. Liu. 1998. Analysis of persistency of lactation calculated from a random regression test day model. Interbull Bull. 17:64-69. 
Kass, R. E., and A. E. Raftery. 1995. Bayes factors. J. Am. Statist. Ass. 90:773-795.

Kirkpatrick, M., D. Lofsvold, and M. Bulmer. 1990. Analysis of the inheritance, selection and evolution of growth trajectories. Genetics 124:979-993.

Leukkunen, A. 1985. Genetic parameters for persistency milk yield in Finnish Ayrshire cattle. Z. Tierzüchtung. Züchtungsbiol. 102:117-124.

Lidauer, M., and E. A. Mäntysaari. 1999. Multiple trait reduced rank random regression test-day model for production traits. Interbull Bull. 22:74-80.

Lidauer, M., E. A. Mäntysaari, I. Strandén, and J. Pösö. 2000. Multiple-trait random regression test day model for all lactations. Interbull Bull. 25:81-86.

Nielsen, U. S., G. A. Pedersen, J. Pedersen, and J. Jensen. 1996. Genetic parameters for mastitis, other diseases and somatic cell count in different parities in Danish dairy breeds. Page 5 in 47th Ann. Mtg. EAAP, Lillehammer, Norway.

Newton, M. A., and A. E. Raftery. 1994. Approximate Bayesian inference with the weighted likelihood bootstrap with discussion. J. R. Statist. Soc. B 56:3-48.

Olori, V. E., W. G. Hill, B. J. McGuirk, and S. Brotherstone. 1999. Estimating variance components for test day milk records by restricted maximum likelihood with a random regression animal model. Livest. Prod. Sci. 61:53-63.

Pryce, J. E., R. F. Veerkamp, R. Thompson, W. G. Hill, and G. Simm. 1997. Genetic aspects of common health disorders and measures of fertility in Holstein Friesian dairy cattle. Anim. Sci. 65:353360 .
Rekaya, R., M. J. Carabaño, and M. A. Toro. 1999. Use of test day yields for genetic evaluation of production traits in HolsteinFriesian cattle. Livest. Prod. Sci. 57:203-217.

Rekaya, R., S. L. Rodriguez-Zas, D. Gianola, and G. E. Shook. 1998. Test-day models for longitudinal binary response: An application to mastitis in Holsteins. Page 45 in 49th Annual Meeting of EAAP, Warsaw, Poland.

Rupp, R., and D. Biochard. 1999. Genetic relationships between clinical mastitis, somatic cell score, production, udder type traits, and milking ease in first lactation Holstein cows. Page 59 in 50th Ann. Mtg. of EAAP, Zurich, Switzerland.

Schaeffer, L. R., and J. C. M. Dekkers. 1994. Random regressions in animal models for test-day production in dairy cattle. Proc. 5th World Congr. Genet. Appl. Livest. Prod. 18:443-446.

Schaeffer, L. R., G. Jamrozik, J. Kistemaker, and J. B. Van Doormaal. 2000. Experience with a test day model. J. Dairy Sci. 83:11351144.

Simianer, H., H. Solbu, and L. R. Schaeffer. 1991. Estimated genetic correlations between disease and yield traits in dairy cattle. J. Dairy Sci. 74:4358-4365.

Uribe, H. A., B. W. Kennedy, S. W. Martin, and D. F. Kelton. 1995 Genetic parameters of common health disorders of Holstein cows. J. Dairy Sci. 78:421-430.

Van der Linde, R. A. Groen, and G. de Jong. 2000. Estimation of genetic parameters for persistency of milk production in dairy cattle. Interbull Bull. 25:113-116.

Van der Werf, J. H. J., M. E. Goddard, and K. Meyer. 1998. The use of covariance functions and random regressions for genetic evaluation of milk production based on test day records. J. Dairy Sci. 81:3300-3308.

Wright, S. 1934. An analysis of variability in number of digits in an inbred strain of guinea pigs. Genetics 19:506-536. 